discovery of new disease mechanisms will undoubtedly accelerate the development of therapies in the treatment of cardiac conduction disorders.

\section{Acknowledgments}

The authors are supported in part by grants from the NIH (HL105983 and HL82727) and NYSTEM (N11G-032) (to G.I. Fishman).

Address correspondence to: Glenn I. Fishman, Leon H. Charney Division of Cardiology, NYU School of Medicine, 522 First Avenue, Smilow 801, New York, New York 10016, USA. Phone: 212.263.3967; Fax: 212.263.3972; E-mail: glenn.fishman@ nyumc.org.

1. Sotoodehnia $\mathrm{N}$, et al. Common variants in 22 loci are associated with QRS duration and cardiac ventricular conduction. Nat Genet. 2010;42(12):1068-1076.

2. Holm H, et al. Several common variants modu-

late heart rate, PR interval and QRS duration. Nat Genet. 2010;42(2):117-122.

3. Pfeufer A, et al. Genome-wide association study of

PR interval. Nat Genet. 2010;42(2):153-159.

4. Chambers JC, et al. Genetic variation in SCN10A influences cardiac conduction. Nat Genet. 2010; 42(2):149-152.

5. Akopian AN, Sivilotti L, Wood JN. A tetrodotoxinresistant voltage-gated sodium channel expressed by sensory neurons. Nature. 1996;379(6562):257-262.

6. Chen Q, et al. Genetic basis and molecular mechanism for idiopathic ventricular fibrillation. Nature. 1998;392(6673):293-296.

7. Probst $\mathrm{V}$, et al. Haploinsufficiency in combination with aging causes SCN5A-linked hereditary Lenegre disease. J Am Coll Cardiol. 2003;41(4):643-652.

8. Papadatos GA, et al. Slowed conduction and ventricular tachycardia after targeted disruption of the cardiac sodium channel gene Scn5a. Proc Natl Acad Sci US A. 2002;99(9):6210-6215.

9. Bezzina CR, et al. Common variants at SCN5ASCN10A and HEY2 are associated with Brugada syndrome, a rare disease with high risk of sudden cardiac death. Nat Genet. 2013;45(9):1044-1049.

10. Yang T, Atack TC, Stroud DM, Zhang W, Hall L, Roden DM. Blocking Scn10a channels in heart reduces late sodium current and is antiarrhythmic. Circ Res. 2012;111(3):322-332.

11. Verkerk AO, et al. Functional Nav1.8 channels in intracardiac neurons: the link between SCN10A and cardiac electrophysiology. Circ Res. 2012;111(3):333-343

12. van den Boogaard $M$, et al. A common genetic variant within $S C N 10 A$ modulates cardiac $S C N 5 A$ expression. J Clin Invest. 2014;124(4):1844-1852.

13. Arnolds DE, et al. TBX5 drives Scn5a expression to regulate cardiac conduction system function. JClin Invest. 2012;122(7):2509-2518.

14. van den Boogaard $M$, et al. Genetic variation in T-box binding element functionally affects SCN5A/SCN10A enhancer. J Clin Invest. 2012; 122(7):2519-2530.

15. Dun W, Boyden PA. Does SCN10A gene product play a role in canine Purkinje cell electrophysiology? Poster presented at: Heart Rhythm 2012: 33rd Annual Scientific Sessions; May 9-12, 2012; Boston, Massachusetts, USA.

16. Facer $\mathrm{P}$, et al. Localisation of SCN10A gene product $\mathrm{Na}(\mathrm{v}) 1.8$ and novel pain-related ion channels in human heart. Int Heart J. 2011;52(3):146-152.

17. Yang T, Atack TC, Abraham RL, Darbar D, Roden DM. Striking electrophysiologic differences between cardiac sodium channel isoforms SCN10A and SCN5A [abstract]. Circulation. 2011;124:A16237.

18. Hu D, et al. Genetic Variants in SCN10A Associated with Brugada Syndrome, Right Bundle Branch Block and Atrioventricular Block. Poster presented at: Heart Rhythm 2012: 33rd Annual Scientific Sessions; May 9-12, 2012; Boston, Massachusetts, USA.

19. Clatot J, et al. Dominant-negative effect of SCN5A $\mathrm{N}$-terminal mutations through the interaction of $\mathrm{Na}(\mathrm{v}) 1.5 \alpha$-subunits. Cardiovasc Res. 2012;96(1):53-63.

20. Qi B, et al. Nav1.8 channels in ganglionated plexi modulate atrial fibrillation inducibility [published online ahead of print February 2, 2014]. Cardiovasc Res. doi:10.1093/cvr/cvu005.

21. Blasius AL, et al. Hypermorphic mutation of the voltage-gated sodium channel encoding gene Scn10a causes a dramatic stimulus-dependent neurobehavioral phenotype. Proc Natl Acad Sci U S A. 2011;108(48):19413-19418.

\title{
Not simply misshapen red cells: multimolecular and cellular events in sickle vaso-occlusion
}

\author{
Gregory M. Vercellotti and John D. Belcher
}

Vascular Biology Center, Division of Hematology, Oncology and Transplantation,

Department of Medicine, University of Minnesota, Minneapolis, Minnesota, USA.

\begin{abstract}
Thromboinflammatory diseases result from the interactions of vascular endothelial cells, inflammatory cells, and platelets with cellular adhesion molecules, plasma proteins, and lipids. Tipping the balance toward a prothrombotic, proinflammatory phenotype results from multicellular activation signals. In this issue of the JCI, Li et al. explore the regulation of heterotypic neutrophil-platelet contacts in response to TNF- $\alpha$-induced venular inflammation with relevance to sickle cell disease (SCD).
\end{abstract}

\section{Not just misshapen red cells}

In a 1910 report of an anemic West Indian man, Herrick first described the "peculiar elongated and sickle-shaped" rbcs produced by individuals with sickle cell anemia (1). Over the next century, evaluation of sickle cell anemia-associ-

Conflict of interest: Gregory M. Vercellotti and John D. Belcher received research funding from Sangart, Inc. and Seattle Genetics.

Citation for this article: J Clin Invest. 2014; 124(4):1462-1465. doi:10.1172/JCI75238. ated molecular and cellular pathobiology revealed that the polymerization of hemoglobin S and cellular shape change upon deoxygenation were due to a single nucleotide mutation (A to $\mathrm{T}$ ) in the gene encoding $\beta$-globin. The pain crises and organ infarctions that manifest in patients were attributed to a mechanical obstruction of blood flow due to the rigid crescent-shaped cells; however, in 1980 Hebbel and others demonstrated that sickle erythrocytes were excessively adherent to vascular endothelial cells (2).
As a result of this seminal observation, researchers in the 1980s and 1990s were able to define the red cell characteristics, endothelial adhesion molecules, and plasma factors responsible for these phenomena. Patients with sickle cell disease (SCD) have marked leukocytosis, thrombocytosis, markers of inflammation, oxidative stress, and a procoagulant phenotype. Other cells have been implicated in sickle-associated vaso-occlusive events, including neutrophils $(3,4)$, monocytes $(5,6)$, platelets $(4,7)$, invariant NKT lymphocytes (8), and the endothelium itself $(2,9-11)$.

\section{Of mice and men: murine SCD models tell a hot story}

Transgenic mouse models of SCD have redefined the pathophysiology of vasoocclusion. SCD models have been developed 


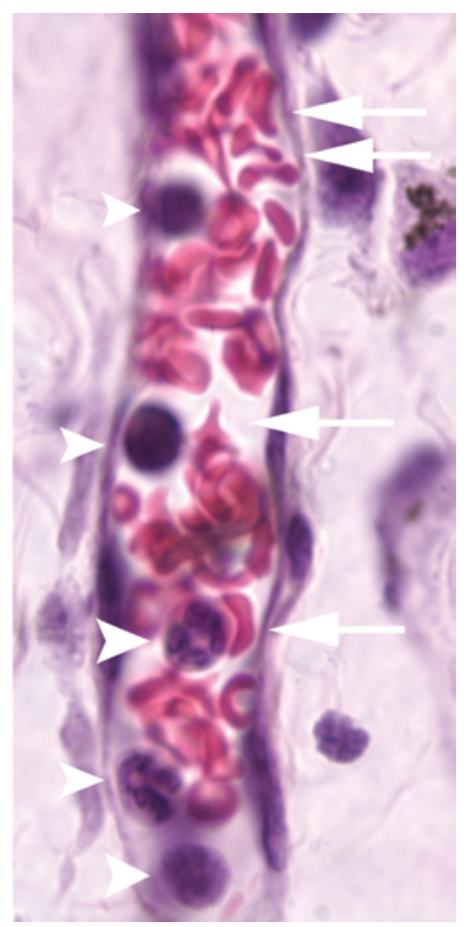

with varying degrees of severity, exhibiting hemolytic anemia, leukocytosis, markers of inflammation, and activated coagulation. Furthermore, these animal models mimic the pathology of human disease in response to stimuli, including hypoxiareoxygenation, cytokines, LPS, and hemoglobin/heme (12-14). Kaul and Hebbel showed that pathological stimuli, such as hypoxia-reoxygenation, resulted in decreased blood flow, enhanced leukocyte rolling, adhesion, emigration, and enhanced oxidant production, strongly supporting the concept that SCD is a disease of inflammation, oxidative stress, and reperfusion/injury physiology (9). Turhan and Frenette demonstrated that leukocytes mediate TNF- $\alpha$-induced vasoocclusion, which leads to reduced blood flow and death. Leukocyte-dependent vaso-occlusion was prevented in mice lacking both P- and E-selectins (3). We have shown that treatment with antibodies targeting multiple adhesion molecules, including VCAM-1, ICAM-1, P-selectin, E-selectin, $\alpha 4 \beta 1, \alpha V \beta 3$, vWF, or PECAM- 1 , inhibits microvascular stasis as assessed with a dorsal skinfold chamber on sickle mice in response to hypoxia-reoxygenation or hemoglobin/heme infusion (11). Histology of a suspected vascular obstruction in the skin of a transgenic sickle mouse following hypoxia-reoxygenation indicates that multiple cell types are present within

\section{Figure 1}

Histology of a venule in the dorsal skin of transgenic sickle mice after 1 hour of hypoxia and 1 hour of reoxygenation. Dorsal skin samples were taken for histological analysis after S+S-Antilles sickle mice were exposed to 1 hour of hypoxia and 1 hour of reoxygenation. Skin samples were fixed overnight in formalin, cut into 5- $\mu \mathrm{m}$ sections, embedded in paraffin, mounted on slides, and stained with hematoxylin and eosin before microscopic examination. The image shows a venule with a suspected vascular obstruction. White arrowheads point to leukocytes that appear to be adherent to the vascular endothelium, and white arrows point to misshapen rbcs inside the venule. Reprinted with permission from American Journal of Hematology (24).

the blockage (Figure 1), and several cellular molecules and interactions have been implicated in murine vaso-occlusion (Figure 2).

Human sickle rbcs and platelets interact with monocytes and neutrophils, which can be found within heterotypic aggregates $(4,6)$. Hemolysis is critical in the vasculopathy of SCD (15), and recent studies demonstrating neutrophil extracellular trap (NET) formation in response to heme further implicate interactions between leukocytes and sickle cells in vaso-occlusion development $(16,17)$. Leukocytosis in SCD patients is associated with an increased risk of acute chest syndrome, stroke, and mortality, adding credence to the hypothesis that SCD is a disease of inflammation.

\section{Don't forget the platelet in SCD}

Most patients with SCD have a high platelet count that is related, in part, to inflammation and the asplenic state. Just as activated leukocytes have been investigated in SCD, so have activated platelets $(4,7)$. Platelets may contribute to vaso-occlusion and stroke in SCD patients due to hemolysis associated with platelet activation via released ADP acting on G proteincoupled purinergic receptor P2Y12 (18) or heme interactions with TLR4 (11). Activated platelets can promote sickle red cell adhesion, form platelet-red cell, plateletneutrophil, and platelet-monocyte aggregates, activate coagulation through CD40 expression, and interact with activated vWF. Platelet aggregates with neutrophils and monocytes result from interactions between platelet P-selectin and P-selectin glycoprotein ligand-1 (PSGL-1) on leukocytes and interactions between platelet glycoprotein Ib $\alpha$ (GPIb $\alpha$ ) and integrin $\alpha \mathrm{M} /$ $\beta 2$ on monocytes. High levels of platelet- monocyte and platelet-neutrophil aggregates have been observed in SCD patients. Recent trials have examined the ability of the P2Y12 inhibitor prasugrel to reduce pain crises (18), and the $\alpha I I b / \beta I I I a$ inhibitor eptifibatide is currently being examined to relieve SCD-associated symptoms (19). Additionally, efficacy of a glycomimetic pan-selectin inhibitor (GMI-1070) is now being tested in SCD patients (20).

\section{How are multicell interactions conducted in this orchestra?}

The complex orchestration that leads to vaso-occlusion in SCD requires one undeniable factor: the presence of hemoglobin S. Recently, several models have been proposed for the events leading to vasoocclusion; however, the pathogenesis of SCD-associated vaso-occlusion remains unclear. If only Raquel Welch and Steven Boyd were available for a fantastic voyage into the vasculature aboard their miniaturized submarine Proteus to record the cellular and molecular events in the hypoxic environment of the post-capillary venules. Is the deoxygenated red cell interacting with the endothelium the first trigger? Or is free hemoglobin/heme acting on the endothelium to blame? Are stiff, misshapen red cells blocking flow? Which proteins, lipids, carbohydrates, and receptors are initially engaged, and how does the crosstalk between cell types proceed? How do neutrophils, monocytes, and platelets aggregate to occlude blood flow? Why is occlusion temporary in most cases, and what signals promote dissolution of the blockade? What factors determine irreversible blockage with subsequent infarction? Why does interference with so many different molecules on cells interfere with vasoocclusion in mouse studies?

In this issue of the JCI, Li et al. (21) focus on the role of AKT, a Ser/Thr kinase that phosphorylates numerous substrates that are important in cell activation, survival, and proliferation. Isoforms of AKT have specific roles in platelets, macrophages, neutrophils, and endothelial cells. Fluorescence intravital microscopic studies revealed that hematopoietic cell AKT2 is critical for neutrophil recruitment and neutrophil-platelet interactions during TNF- $\alpha$-induced vascular inflammation in live mice. Using in vitro reconstituted systems, Li et al. revealed that both platelet and neutrophil AKT2 are important for heterotypic cell-cell aggregation under shear (21). Basal phosphorylation levels 


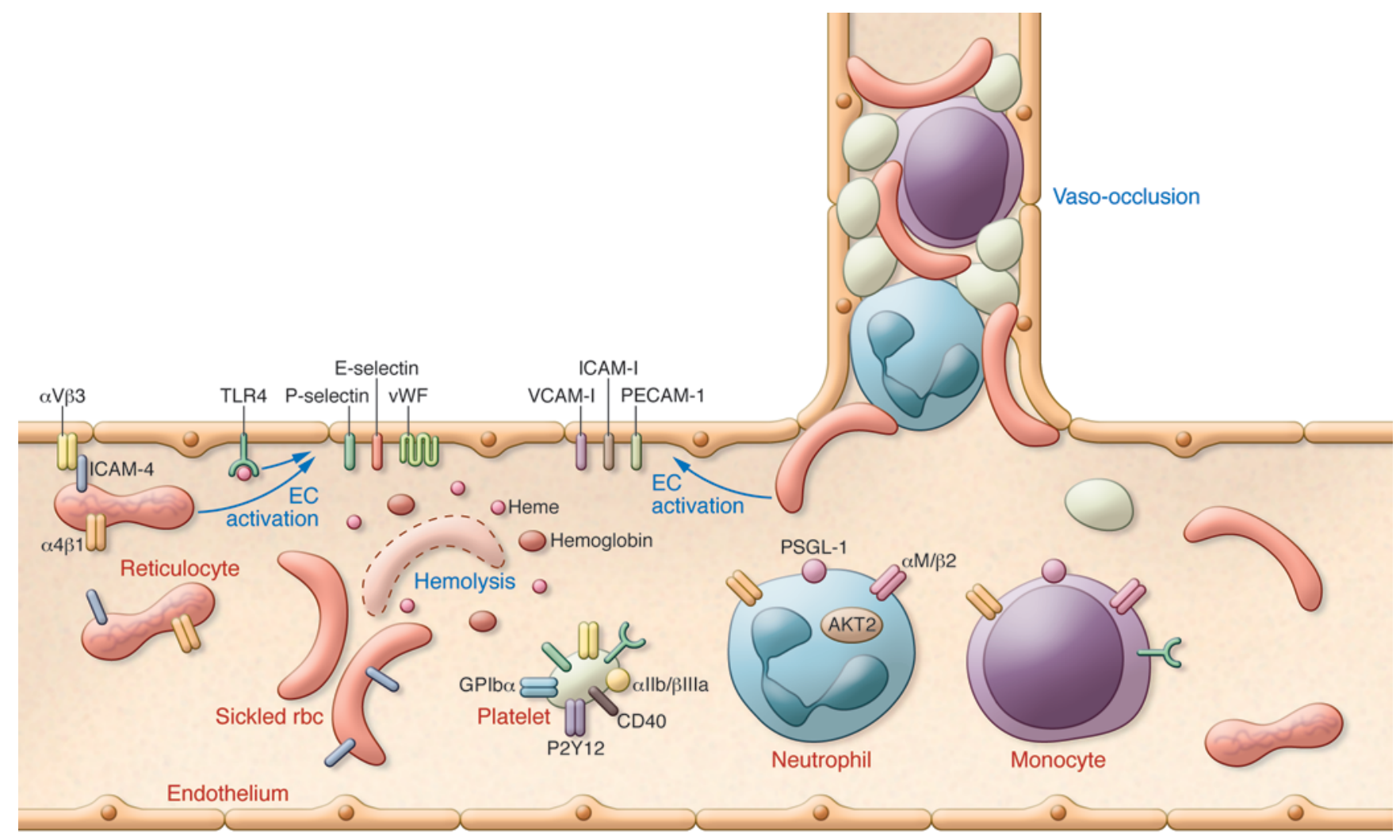

Figure 2

Conceptual model of molecular and cellular interactions that influence the development of vaso-occlusion in SCD mice. Blockade or deletion of factors, including P- and E-selectin, VCAM-1, ICAM-1, and PECAM-1, which are associated with endothelial cell activation, prevents vaso-occlusion in SCD mice. Furthermore, molecules present on platelets, reticulocytes, sickled rbcs, neutrophils, and monocytes have been implicated in the development of murine SCD-associated vaso-occlusion. Li and colleagues (21) have determined that neutrophil AKT2 may be required for vaso-occlusion. Despite the identification of a myriad of molecules and cell types that mediate vaso-occlusion, the timing of events and the initiating signals remain to be determined.

of AKT isoforms were markedly increased in neutrophils and platelets isolated from SCD patients. Inhibition of AKT2 reduced heterotypic aggregation of patients' neutrophils and platelets in vitro and diminished neutrophil adhesion and neutrophil-platelet aggregation in SCD mice, thereby improving blood flow rates. These results provide important evidence that neutrophil-associated AKT2 regulates $\alpha \mathrm{M} / \beta 2$ integrin (CD18/CD11b) function and promotes neutrophil recruitment and neutrophil-platelet interactions in response to thromboinflammatory conditions such as SCD.

This elegant study by $\mathrm{Li}$ and colleagues does not, however, answer the question of who conducts the orchestra of vaso-occlusion, but it provides important insights into the players. Furthermore, the study does not reconcile previous investigations that have demonstrated the importance of P-selectin in vaso-occlusion. Is AKT2 the bad sibling? Caution should be used before targeting AKT2 for SCD therapy. Patients with SCD are prone to infection, and inhibition of AKT could worsen this by inactivating host-defending neutrophils. Mice lacking Akt2 present with hyperinsulinemia and diabetes-like syndrome, because AKT2 is highly expressed in insulin-responsive tissues (22). Previously, the observed phosphorylation of nuclear AKT in livers of sickle mice overexpressing heme oxygenase-1 (HO-1) or after treatment with inhaled $\mathrm{CO}$ suggested an antiinflammatory role for AKT (23). The observed AKT phosphorylation induced by $\mathrm{HO}-1$ and $\mathrm{CO}$ could possibly be due to selective activation of AKT isoforms AKT1 or AKT3.

Ultimately, the primacy of hemoglobin $\mathrm{S}$, hemolysis, oxidative stress, and subsequent inflammation in SCD pathogenesis rules any approaches to limiting SCDassociated damage. Strategies aimed at minimizing hemoglobin $S$, through gene therapy or stem cell transplantation, and minimizing hemoglobin S polymerization, by increasing hemoglobin $\mathrm{F}$, are the best hopes for minimizing SCD patient morbidity and mortality. Approaches geared toward dealing with hemolysis, either by scavenging hemoglobin/heme or detoxifying heme through HO-1, may limit the inflammation. Other strategies for ameliorating SCD, including antiinflammatories, vasoprotectants, and antithrombotics, will be useful; however, these approaches could compromise host defenses. Herrick never could have imagined over 100 years ago the complexity of the pathobiology of the peculiar-shaped cells that he observed. Hopefully, the use of nanotechnology to provide a "miniature submarine" to explore the vasculature of SCD individuals will be on the horizon to provide us with a better understanding of vaso-occlusion.

\section{Acknowledgments}

This work was supported by an NIH grant from the NHLBI (HL115467-01, to G.M. Vercellotti and J.D. Belcher). 
Address correspondence to: Gregory M. Vercellotti, University of Minnesota, 420 Delaware St. SE, MMC 480, Minneapolis, Minnesota 55455, USA. Phone: 612.626.3757; Fax: 612.625.6919; E-mail: verce001@umn.edu.

1. Herrick JB. Peculiar elongated and sickle-shaped red blood corpuscles in a case of severe anemia. Arch Intern Med. 2010;6(5):517-521.

2. Hebbel RP, Yamada O, Moldow CF, Jacob HS, White JG, Eaton JW. Abnormal adherence of sickle erythrocytes to cultured vascular endothelium: possible mechanism for microvascular occlusion in sickle cell disease. J Clin Invest. 1980;65(1):154-160.

3. Turhan A, Weiss LA, Mohandas N, Coller BS, Frenette PS. Primary role for adherent leukocytes in sickle cell vascular occlusion: a new paradigm. Proc Natl Acad Sci U S A. 2002;99(5):3047-3051.

4. Hidalgo A, Chang J, Jang JE, Peired AJ, Chiang EY, Frenette PS. Heterotypic interactions enabled by polarized neutrophil microdomains mediate thromboinflammatory injury. Nat Med. 2009;15(4):384-391.

5. Belcher JD, Marker PH, Weber JP, Hebbel RP, Vercellotti GM. Activated monocytes in sickle cell disease: potential role in the activation of vascular endothelium and vaso-occlusion. Blood. 2000;96(7):2451-2459

6. Brittain JE, Knoll CM, Ataga KI, Orringer EP, Parise LV. Fibronectin bridges monocytes and reticulocytes via integrin $\alpha 4 \beta 1$. Br J Haematol. 2008;141(6):872-881.

7. Brittain HA, Eckman JR, Swerlick RA, Howard RJ,
Wick TM. Thrombospondin from activated platelets promotes sickle erythrocyte adherence to human microvascular endothelium under physiologic flow: a potential role for platelet activation in sickle cell vaso-occlusion. Blood. 1993;81(8):2137-2143.

8. Wallace KL, Linden J. Adenosine A2A receptors induced on iNKT and NK cells reduce pulmonary inflammation and injury in mice with sickle cell disease. Blood. 2010;116(23):5010-5020.

9. Kaul DK, Hebbel RP. Hypoxia/reoxygenation causes inflammatory response in transgenic sickle mice but not in normal mice. J Clin Invest. 2000;106(3):411-420.

10. Zennadi R, et al. Erythrocyte plasma membranebound ERK1/2 activation promotes ICAM-4-mediated sickle red cell adhesion to endothelium. Blood. 2012;119(5):1217-1227.

11. Belcher JD, et al. Heme triggers TLR4 signaling leading to endothelial cell activation and vasoocclusion in murine sickle cell disease. Blood. 2014;123(3):377-390

12. Fabry ME, et al. High expression of human beta S- and alpha-globins in transgenic mice: erythrocyte abnormalities, organ damage, and the effect of hypoxia. Proc Natl Acad Sci U S A. 1992;89(24):12155-12159.

13. Paszty C, et al. Transgenic knockout mice with exclusively human sickle hemoglobin and sickle cell disease. Science. 1997;278(5339):876-878.

14. Wu LC, Sun CW, Ryan TM, Pawlik KM, Ren J, Townes TM. Correction of sickle cell disease by homologous recombination in embryonic stem cells. Blood. 2006;108(4):1183-1188.

15. Kato GJ, Hebbel RP, Steinberg MH, Gladwin MT. Vasculopathy in sickle cell disease: biology, pathophysiology, genetics, translational medi- cine, and new research directions. Am J Hematol. 2009;84(9):618-625.

16. Fuchs TA, Wagner DD, Frenette PS. Heme-induced neutrophil extracellular traps (NETs) formation contributes to sickle cell disease pathogenesis. Blood. 2013;122(21):184.

17. Mathson K, Teena R, Belcher JD, Vercellotti GM, Slungaard A. Thiocyanate blocks peroxidasedependent extracellular trap (ET) formation by PMN and eosinophils: heme is a potent new agonist for the ET pathway. Blood. 2013;122(21):323.

18. Jakubowski JA, et al. A phase 1 study of prasugrel in patients with sickle cell disease: effects on biomarkers of platelet activation and coagulation. Thromb Res. 2014;133(2):190-195.

19. Desai PC, et al. A pilot study of eptifibatide for treatment of acute pain episodes in sickle cell disease. Thromb Res. 2013;132(3):341-345.

20. Chang J, Patton JT, Sarkar A, Ernst B, Magnani JL, Frenette PS. GMI-1070, a novel pan-selectin antagonist, reverses acute vascular occlusions in sickle cell mice. Blood. 2010;116(10):1779-1786.

21. Li J, et al. Neutrophil AKT2 regulates heterotypic cell-cell interactions during vascular inflammation. J Clin Invest. 2014;124(4):1483-1496.

22. Cho $\mathrm{H}$, et al. Insulin resistance and a diabetes mellitus-like syndrome in mice lacking the protein kinase Akt2 (PKB $\beta$ ). Science. 2001;292(5522):1728-1731.

23 . Belcher JD, et al. Heme oxygenase-1 gene delivery by Sleeping Beauty inhibits vascular stasis in a murine model of sickle cell disease. J Mol Med (Berl). 2010;88(7):665-675

24. Kalambur VS, et al. Microvascular blood flow and stasis in transgenic sickle mice: utility of a dorsal skin fold chamber for intravital microscopy. Am J Hematol. 2004;77(2):117-125.

\title{
Insulin, osteoblasts, and energy metabolism: why bone counts calories
}

\author{
Ryan C. Riddle and Thomas L. Clemens \\ Department of Orthopaedic Surgery, Johns Hopkins University School of Medicine, Baltimore, Maryland, USA. \\ Baltimore Veterans Administration Medical Center, Baltimore, Maryland, USA.
}

\begin{abstract}
Recent studies have demonstrated that insulin stimulates bone cells to produce and activate osteocalcin, an endocrine hormone that increases the efficiency of glucose metabolism through its actions on the pancreas and other peripheral tissues. In this issue of the JCI, Wei and colleagues directly explore the contribution of insulin signaling in osteoblasts to the disturbances in whole-body glucose metabolism associated with a high-fat diet. In mice fed a high-fat diet, increased uptake of saturated fatty acids by the osteoblast accelerates the ubiquitination and degradation of the insulin receptor. In this setting, impairments in osteoblast insulin signaling reduce serum levels of undercarboxylated osteocalcin, which in turn exacerbate insulin resistance in muscle and white adipose tissue. These findings underscore the importance of insulin-responsive skeletal cells as components of a newly appreciated endocrine network critical for regulating global energy homeostasis.
\end{abstract}

Conflict of interest: The authors have declared that no conflict of interest exists.

Citation for this article: J Clin Invest. 2014; 124(4):1465-1467. doi:10.1172/JCI75554.

\section{Bone as a metabolic organ: lessons from evolution}

The evolution of a large appendicular skeleton powered by robust skeletal muscles in early tetrapods was a successful strategy for ambulation on land. Additionally, the new skeleton served as a repository for calcium, a scarce commodity in the terrestrial habitat, and the emergence of the parathyroid gland at this juncture provided the means to rapidly access bone calcium through osteoclast-mediated liberation from skeletal stores (1). However, the upgraded musculoskeletal system also increased overall fuel requirements and altered global energy balance, prompting the evolution of other endocrine networks to coordinate energy expenditure (2). Prominent among these emerging networks were the leptin and insulin/insulin-like growth factor pathways, which have assumed central roles in growth and metabolism in higher organisms $(3,4)$. The importance of these meta- 